\title{
Physics of alpha channelling and related TFTR experiments
}

\author{
N.J. Fisch \\ Princeton Plasma Physics Laboratory, Princeton University, \\ Princeton, New Jersey, United States of America
}

\begin{abstract}
Over the last thirty years, there have been increasingly ambitious theories and experiments along the theme of exercising increased control over plasma by means of RF waves. The diversion of energy from energetic alpha particles to waves (alpha channelling) is such an attempt at detailed control over plasma behavior. The effect could accelerate progress towards an economical deuteriumtritium tokamak reactor. Recent simulations and experiments on TFTR support certain separate building blocks that taken together might produce the desired effect.
\end{abstract}

\section{Introduction}

Over the last thirty years, microwaves have been put to use for more and more precise control of tokamak plasma. This detailed control of charged particles in a plasma, in both velocity and configuration space, is what might be called 'phase space engineering' by microwaves. As we built upon our knowledge of microwaves, our attempts at phase space engineering grew more ambitious. This article provides a historical perspective on these attempts. It also provides a perspective on recent experimental data relevant to one of the more recent and more ambitious attempts at phase space engineering, alpha channelling.

In the 1960s and the 1970s, the worldwide effort in $\mathrm{RF}$ waves in magnetic fusion research centred on attaining plasmas close to thermonuclear conditions. Of particular interest was the heating of the plasma to thermonuclear temperatures, say, to at least $10 \mathrm{keV}$. There was great success in advancing microwave heating of plasma in several frequency regimes, including the ion cyclotron frequency regime (10-100 MHz), the lower hybrid frequency regime (1$10 \mathrm{GHz}$ ) and the electron cyclotron frequency regime (100 GHz).

In the late 1970 s and in the 1980s the focus of research into microwaves in tokamaks shifted from heating the plasma to driving toroidal current in the plasma. It was recognized that microwaves were suited for far more detailed control over the plasma behaviour than the mere heating of the plasma. Through proper phasing of the waves, groups of electrons or ions could be targeted through resonance conditions. The waves might deliver momentum to these particles, or affect them in other ways, such as to cause the generation of toroidal current [1]. Current drive in tokamaks enjoyed great and widespread success, particularly when RF current generation was practised by lower hybrid waves [2]. The lower hybrid current drive effect was demonstrated first on small tokamaks, including the Versator, WT-2 and PLT tokamaks. Up to $3 \mathrm{MA}$ have been driven by lower hybrid waves on the JT-60 tokamak.

With confidence in the underpinnings of the theoretical description of current drive processes and in the detailed control exercisable by waves, attention turned in the late 1980 s and the 1990 s to exercising even greater control over the plasma through the application of intense microwaves. One important idea was to exercise control over the radial profile of the plasma current driven by microwaves. Carefully phased microwaves might be damped in a narrow radial region of the tokamak, thus driving current over a narrow regime. Such control over the current profile could produce more attractive MHD equilibria. Other ideas included the excitation of microwaves to create localized regions of ponderomotive force, producing localized transport barriers. Further ideas yet envisage the stabilization of tearing or sawtooth instabilities by localized currents.

Indeed, now, at the end of the 1990s, as we ponder in what directions tokamak research will move in the next millenium, it is noteworthy that the new tokamaks being built, namely KSTAR in Korea [3], SST1 in India [4] and HT-7U in China [5], are all relatively small, superconducting, very long pulse devices in which the plasma current is sustained through lower hybrid current drive. The larger superconducting device, Tore Supra, is planning an upgrade that will accommodate 15 min lower hybrid driven discharges [6]. The superconducting tokamak TRIAM $1 \mathrm{M}$ has already shown that the plasma current can be so sustained for two hours [7]. Researchers at other existing tokamaks, such as Alcator C-Mod 
at MIT [8], are also modelling and planning significantly longer discharges driven by lower hybrid waves. What we will learn from these long pulse tokamaks is how the detailed control exercisable by waves operates on longer timescales, long enough for the plasma radial current profile to relax, or long enough to recognize slow instabilities.

At the same time that we pursue the logical consequences of steady state driven currents, we should keep in mind the larger lessons learned in our ability to engineer the phase space of the plasma. In Section 2, these larger lessons are reviewed. In Section 3, we describe the physics of alpha channeling. In Section 4, we point out the implications of the TFTR experiments related to the alpha channelling effect. In Section 5, we discuss our conclusions.

\section{Lessons from the early current drive campaigns}

The current drive campaign of the 1980s not only demonstrated the current drive effect. It also showed that very detailed control over the plasma phase space could be exercised by waves in a manner predictable by theory. A feature of the theory is that the current drive effect is described in considerable microscopic detail, rather than macroscopic detail; for example, the efficiency for the RF current drive effect was written as a function of velocity space, rather than as an integrated quantity as in the Spitzer resistivity. Moreover, not only was the theory detailed, but there was such detailed agreement between theory and experiment that the assumptions of the theory could be considered verified.

The salient properties of driven plasmas, such as the current drive efficiency, are characterized theoretically by Green's functions, which are functions of velocity space. Moreover, by means of resonance conditions, the RF induced fluxes are localized quantities. For example, a narrow spectrum of lower hybrid waves localized the parallel velocity associated with the wave driven fluxes. If the waves were to resonate with the tail of the electron distribution, then the perpendicular velocity could be ignored compared with the parallel velocity. Moreover, the direction of the flux is known from the nature of the damping and the wave characteristics; the lower hybrid wave drives parallel fluxes in velocity space, whereas the electron cyclotron wave drives nearly perpendicular fluxes. Finally, the magnitude of the driven flux drops out when the steady state current drive efficiency is expressed as a ratio,

$\frac{J_{R F}}{P_{d}}=\frac{\boldsymbol{S} \cdot(\partial / \partial \boldsymbol{v}) \chi(\boldsymbol{v})}{\boldsymbol{S} \cdot(\partial / \partial \boldsymbol{v}) \epsilon(\boldsymbol{v})}$

where $\boldsymbol{S}$ is the RF induced flux, $\chi(\boldsymbol{v})$ is the Green's function for the current drive, $\boldsymbol{v}$ is the velocity of the resonant electrons, i.e. the vicinity in velocity space of the induced flux, and $\epsilon(\boldsymbol{v})=m v^{2} / 2$ is the kinetic energy of the resonant electrons.

The response function $\chi(\boldsymbol{v})$ characterizes the current drive efficiency for each point in velocity space. The RF conductivity is thus a far more detailed description of the plasma than the Spitzer conductivity, which is merely an integrated quantity. For $\mathrm{RF}$ current drive, certain regions of velocity space were identified as most efficient for current drive; by phasing waves suitably, exactly these regions could be accessed experimentally.

Such detail in the theory and the possibility of precise phasing of the waves permitted a very detailed comparison between theory and experiment. Over 300 shots of PLT, in several regimes of current drive, including current rampup, steady state and current rampdown, matched theoretical predictions with remarkable accuracy [9]. Such a close check of the theory really did much more than just to demonstrate the current drive effect. It verified closely held beliefs about the nature of the current carrying superthermal electrons that were the key to forming the theory of current drive in the first place, namely that superthermal electrons slowed down classically by Coulomb collisions and interacted quasi-linearly with waves.

Note that such knowledge could not have been taken for granted in the 1980s. There was no guarantee that superthermal electrons would not be subject to some anomalous slowing down mechanism, say due to plasma turbulence. Such an effect would not have been detected by measurements of plasma conductivity, which would only give integrated quantities, which would reflect just the properties of the bulk electrons. It took the detailed control over the plasma, exercised in the current drive campaigns of the 1980s, to demonstrate the underpinnings of the theory on which the current drive effect was based.

The agreement between theory and experiment also allows greater confidence in the possibility of detailed manipulation of the plasma through microwaves. Through resonance conditions, select groups of particles can, in fact, be diffused in phase space along precise directions. More and more 
ambitious attempts at phase space engineering might then be contemplated.

Indeed, in addition to diffusing along specified directions, specified groups of the plasma electrons and fuel ions, RF waves might also be used to diffuse with precision the by-products of the DT fusion reaction, namely, the alpha particles. The idea of 'alpha channelling' involves the use of microwaves to diffuse alpha particles to lower energy, releasing their energy to the microwaves even as the cooled alpha particles would be transported to the tokamak periphery. With carefully chosen microwave parameters and by means of resonance conditions, the microwaves are used to manipulate in detail the particle phase space, selecting certain particles for diffusion along certain paths in both velocity space and real space.

\section{Alpha channelling effect}

The alpha channelling effect involves the diverting or channelling of energy from energetic alpha particles to ions via waves. Convectively amplified waves collisionlessly diffuse alpha particles to lower energy, growing at the expense of the alpha particle energy. Waves are a useful form of energy that might then maintain the departure from equilibrium. Since the waves are amplified at the expense of the alpha particles, the higher reactivity would then be reached without substantial external power. The alpha particle power is thus effectively 'channelled' into a more useful form of power.

The redirection of power is particularly useful in the case of maintaining the hot ion mode, since, in the absence of the channelling effect, the alpha particle power naturally flows mainly into the electrons, making them always hotter than the ions. Power channelled to waves, however, could be damped on ions, at once reducing the electron heating and increasing the ion heating. This makes $T_{i}>T_{e}$ possible, conserving the fuel pressure for the ions. If the alpha particle power is collisionally damped on electrons, the hot ion mode cannot be reached.

The free energy of the alpha particles is best tapped by exploiting the population inversion along a path in both energy and space [10]. The channelling occurs when the diffusion path connects high energy alpha particles at the plasma centre to low energy alpha particles at the plasma periphery. When one wave is utilized, there can be only one diffusion path, with stringent constraints on the alpha particle motion. The alpha particle motion is constrained to lie on a one dimensional curve, a line. If the path is chosen appropriately, then, if an alpha particle gains energy, it must diffuse to the tokamak periphery; conversely, if it loses energy in interacting with the wave, it must diffuse to the tokamak centre. Since alpha particles exit only at the periphery, eventually they are diffused by the wave to the tokamak periphery where they give up a precise amount of energy to the wave which is proportional to their distance travelled in reaching the periphery.

The effect can be seen most easily in a slab with periphery at $x=a$ and 'centre' at $x=0$, so that particles can escape only at $x=a$. (Here, $x$ plays the role of the minor radius $r$ in a tokamak.) Consider a magnetic field in the $z$ direction, with alpha particles exchanging energy with waves travelling in the $y$ direction. The ratio of displacement of the guiding centre in the $x$ direction, $\Delta x$, to (perpendicular) energy absorbed, $\Delta E$, is

$\Delta x=-k_{y} \Delta E / m \Omega \omega$.

This quantity is determined by wave and particle parameters only; $\omega$ is the wave frequency, $k_{y}$ is the wavenumber in the $y$ direction, $m$ is the alpha particle mass and $\Omega \equiv 2 e B / m$ is the alpha particle gyrofrequency. Upon repeated interactions with the wave, an alpha particle will trace a line in $x-E$ space.

For efficient channelling, one would then require $\Delta x / \Delta E \sim a / E_{\alpha}$, where $E_{\alpha}$ is the alpha particle birth energy, 3.5 MeV. If instead $\Delta x / \Delta E \gg a / E_{\alpha}$, then the alpha particle would be extracted from the centre with almost all its energy intact, whereas if $\Delta x / \Delta E \ll a / E_{\alpha}$, then the alpha particles are not extractable from the plasma centre. In this case, a population inversion is not likely to occur, and the wave will not be amplified.

Toroidal geometry is more complicated than slab geometry, but the considerations are similar. Particles interacting with one wave trace a straight line in $\epsilon-\mu-P_{\phi}$ space, where $\mu=m v_{\perp}^{2} / 2 B$ is the magnetic moment, $\epsilon=\mu B+m v_{\|}^{2} / 2$ is the kinetic energy and $P_{\phi}=R\left(m B_{\phi} v_{\|} / B-q A_{\phi}\right)$ is the canonical angular momentum, with $A_{\phi}$ being the vector potential. Each point in $\epsilon-\mu-P_{\phi}$ space represents a single guiding centre orbit for trapped particles, and, for each sign of $v_{\|}$, a passing particle orbit. Given $\epsilon, \mu$ and $P_{\phi}$, and the sign of $v_{\|}$for passing orbits, it may be determined if the orbit intersects the plasma periphery, thus losing the particle.

Upon interaction with a single wave with toroidal mode number $n_{\phi}$, and absorbing energy $\Delta \epsilon, P_{\phi}$ changes by

$\Delta P_{\phi}=\left(n_{\phi} / \omega\right) \Delta \epsilon$. 
Assume that the exchange of energy occurs only for particles satisfying the resonance condition

$\omega-k_{\|} v_{\|}=n \Omega$

where $n$ is an integer, then, upon absorbing energy $\Delta \epsilon, \mu$ changes by

$\Delta \mu=(n Z e / m \omega) \Delta \epsilon$

where $e$ is the electron charge, and, for alpha particles, $Z=2$. Thus, upon repeated interaction with one wave, the constants of motion, $\epsilon, \mu$ and $P_{\phi}$, trace a straight line.

It turns out that achieving the alpha channelling effect with one wave only is probably too difficult it is hard to find waves with precisely the right characteristics. However, the effect appears to be achievable using two waves instead of one wave, one wave to break the adiabatic $\mu$ invariant and one wave to move particles large distances without energy extraction [11]. The mode converted ion Bernstein wave (IBW) will break the invariance of $\mu$ and thereby access the particle's perpendicular energy. A low frequency, such as the toroidal Alfvén eigenmode (TAE) preserves $\mu$, while moving the alpha particles large distances with little energy exchange.

But while one wave diffuses along a line, several waves diffuse along a web in $\epsilon-\mu-P_{\phi}$ space. Note that, in the case of one wave, if the alpha particle leaves at the periphery through collisionless diffusion, it must necessarily be cooled and the wave must necessarily acquire the particle's energy. For two waves, these theorems no longer hold. However, by selectively choosing where the diffusion paths exist in phase space (by means of resonance conditions and spatial localization of the waves), configurations of two waves might be created so that the alpha particles are strongly predisposed to lose energy to the waves [12].

\section{TFTR experiments}

The assertion that configurations of waves might be created to channel alpha particle power to waves is based on numerical simulations [12]. These simulations showed that by using two waves, the IBW and the TAE, two thirds of the energy might be extracted from the ejected alpha particles (90\% of the total birth distribution) in a reactor. There is still the issue of coupling the wave energy for useful purposes. But there are still more optimizations to be done, so the substantial energy extraction even without further optimization is promising. These simulations rely, however, on several assumptions concerning the wave physics, including assumptions about IBW wave propagation and quasi-linear damping of the IBW.

Note that the basic propagation theory of the IBW and the TAE has been experimentally verified, but our concern now is the IBW experiments. Experiments on TFTR demonstrated significant control in positioning the IBW [13], as well as significant interactions with fast ions [14]. Here, however, attention is drawn to two basic and key, but subtle, assumptions. These two assumptions are derived with great certainty, but require experimental verification, for which data on TFTR were highly informative. One assumption, the so-called ' $k_{\|}$flip', was clearly verified on TFTR. The other assumption, quasi-linear damping of the IBW wave, is still quite a puzzle, although a puzzle that appears to have some very promising features.

The $k_{\|}$flip, which had been predicted theoretically [15], occurs as follows: as the IBW emerges from the mode conversion layer, there is a rapid increase, as a function of horizontal position, in $k_{x}$, the perpendicular wavenumber in the direction of the magnetic field gradient (here, the horizontal or $\hat{x}$ direction). Since the poloidal magnetic field has a component in the $\hat{x}$ direction, the parallel wavenumber can be written as $k_{\|}=n_{\phi} / R+k_{x} \hat{\boldsymbol{x}} \cdot \hat{\boldsymbol{B}}$, where $n_{\phi} / R$ is the launched $k_{\|}$and $\hat{B}$ is the direction of the magnetic field. Note that either above or below the midplane $k_{\|}$may change sign from that of the launched $k_{\|}$.

The flip is critical for two reasons: first, in flipping, the parallel phase velocity becomes infinite, so electron damping can be avoided. Second, alpha particles will cool as they leave the plasma only for $n_{\phi}>0$. From the resonance condition, we have $v_{\|}=\left(\omega-\Omega_{\alpha}\right) / k_{\|}>0$. Since mode conversion in DT plasmas occurs to the high field side of the deuterium gyroresonance layer, $\omega<\Omega_{\alpha}$. Thus, where the wave-particle interaction occurs, $k_{\|}$must be negative, which is necessarily opposite in sign to the launched $k_{\|}$.

In TFTR, in a $\mathrm{D}^{3} \mathrm{He}$ plasma, large beam losses occurred when co-going deuterium beams were injected along with IBWs phased in the counterstreaming direction, but not in the co-streaming direction [16]. In a $\mathrm{D}^{3} \mathrm{He}$ plasma, the deuterium resonance is on the high field side of the mode conversion layer, so $\omega>\Omega_{D}$. Thus, to affect co-going particles, $k_{\|}>0$, which is opposite in direction to $n_{\phi}$, hence 'flipped'. This is verification of an aspect of 
linear wave propagation that is not surprising, even if the effect appears somewhat unusual. Its verification, however, if not surprising, is still reassuring, because of the importance of the effect to the use of the IBW for alpha channelling.

The second assumption about which TFTR has been very informative concerns the quasi-linear theory of the IBW and fast ions. A very rough estimate, assuming wave propagation of the IBW wave with quasi-linear diffusion of alpha particles based on uncorrelated kicks by the IBW, predicts that about $100 \mathrm{MW}$ of power is necessary to produce a large enough quasi-linear diffusion of alpha particles that they will slow down collisionlessly rather than collisionally [17]. This is the so-called 'collisionless limit'. Note that for a $2 \mathrm{GW}$ (electric) reactor, there is $400 \mathrm{MW}$ of alpha particle power, so $100 \mathrm{MW}$ is a tolerable amount of $\mathrm{RF}$ power to recirculate to attain the collisionless limit. But several times this number would not be tolerable economically.

Now in TFTR, deuterium beams with a duration of only $50 \mathrm{~ms}$ were fired into discharges with varying amounts of IBW power [18]. The data, composed from the detection of heated beam ions at the periphery, are quite rich in detail; in addition to the poloidal angle, energy, and pitch angle of the exiting ions, there is now the time from the blip of the ions striking the detector. All these four dimensional data can be gathered as a function of RF power.

The detected losses as a function of power turn on at a threshold power, and then should level off beyond a second characteristic power. Both characteristic powers are related to overcoming collisional slowing down. Indeed, this is what is seen both in simulations [17] and in the TFTR experiment [16].

But it is remarkable that in order to obtain the simulation to turn on at about the same threshold power as the experiment, the effective diffusion by waves needed to be increased in the simulation by 40 70 compared with what simple quasi-linear theory would suggest $[17,19]$. This is not easy to explain. It means that the diffusion caused by the waves is much larger than we thought it could be. That is a discrepancy that cannot be explained by the power not coupling properly to the plasma; on the contrary, the power is being utilized too effectively. Note that the level of detail in both the theory and the experiments is large, so that it would not be possible to confuse ripple trapped beam deuterons escaping at $80 \mathrm{keV}$ at the $20^{\circ}$ detector with deuterons escaping at the $60^{\circ}$ detector after being heated to $2.2 \mathrm{MeV}$ through quasi-linear diffusion by the IBW. This means that easy answers for explaining the TFTR data may be hard to come by.

This may mean that there are strong correlations that either invalidate quasi-linear theory or yield a different diffusion coefficient. Possibly it means that there is some high undamped internal eigenmode that is ringing with the excited IBW. In any event, the fundamental physics is, at present, in need of improvement. The fact that the effective diffusion by waves is tens of times larger than expected means that, should the effect scale to a reactor, the collisionless regime for effective channelling could be achieved at almost negligible circulating power.

\section{Summary}

To summarize, microwaves have been put to increasingly more sophisticated uses over the last thirty years. As greater control is exercised over the plasma, there is greater opportunity also to check the fundamental principles underlying the physics of microwaves in plasma. Thus, the current drive campaign gave us, in addition to steady state operation, confidence in our representation of the physics of resonant superthermal electrons.

In examining recent TFTR data relevant to the alpha channelling effect, again there is a comparison that can be made between the detailed data available in the lost ion detectors and the detailed theory of resonant particle diffusion in $\epsilon-\mu-P_{\phi}$ space. Although certain data concerning the linear wave propagation of the IBW do agree with theoretical predictions, including the important $k_{\|}$flip, there are huge and interesting discrepancies concerning the quasi-linear diffusion coefficient.

Just as in the case of current drive response functions, there is much detail here both in the theory and in the experimental observables. In trying to produce the alpha channelling effect there is as much to be learned about low frequency waves interacting with ions as there was to be learned about the dynamics of superthermal electrons in trying to produce the lower hybrid current effect. However, at the present time, our understanding of the details of the ion dynamics appears to be not at all as complete as one might have imagined.

\section{Acknowledgements}

Much of the review presented here is based on work of the author and his colleagues. The influence 
on the present work of the author's long term collaborators, C.F.F. Karney, J.-M. Rax and M.C. Herrmann, is gratefully acknowledged. This work was supported by the USDOE under Contract No. DEAC02-76-CHO3073.

\section{References}

[1] Fisch, N.J., Rev. Mod. Phys. 59 (1987) 175.

[2] Fisch, N.J., Phys. Rev. Lett. 41 (1978) 873.

[3] Lee, G.S., paper presented at TCM on Steady-State Operation of Magnetic Fusion Devices, Fukuoka, 1999.

[4] Saxena, Y.C., Nucl. Fusion 40 (2000) 1069.

[5] Yuanxi Wan, Nucl. Fusion 40 (2000) 1057.

[6] Equipe Tore Supra, Nucl. Fusion 40 (2000) 1047.

[7] Itoh, S., et al., in Fusion Energy 1996 (Proc. 16th Int. Conf. Montreal, 1996), Vol. 1, IAEA, Vienna (1997) 351.

[8] Bonoli, P.T., Nucl. Fusion 40 (2000) 1251.

[9] Karney, C.F.F., Fisch, N.J., Jobes, F.C., Phys. Rev. A 32 (1985) 2554.

[10] Fisch, N.J., Rax, J.M., Phys. Rev. Lett. 69 (1992) 612.

[11] Fisch, N.J., Herrmann, M.C., Nucl. Fusion 35 (1995) 1753.
[12] Herrmann, M.C., Fisch, N.J., Phys. Rev. Lett. 79 (1997) 1495.

[13] Majeski, R., et al., in Plasma Physics and Controlled Nuclear Fusion Research 1994 (Proc. 15th Int. Conf. Seville, 1994), Vol. 1, IAEA, Vienna (1995) 443.

[14] Darrow, D.S., et al., Phys. Plasmas 3 (1996) 1875.

[15] Valeo, E.J., Fisch, N.J., Phys. Rev. Lett. 73 (1994) 3536.

[16] Fisch, N.J., et al., in Fusion Energy 1996 (Proc. 16th Int. Conf. Montreal, 1996), Vol. 1, IAEA (1997) 271.

[17] Herrmann, M.C., PhD Thesis, Princeton Univ. (1998).

[18] Darrow, D.S., et al., Nucl. Fusion 36 (1996) 509.

[19] Fisch, N.J., Herrmann, M.C., Plasma Phys. Control. Fusion 41 (1999) A221.

(Manuscript received 30 November 1999

Final manuscript accepted 24 February 2000)

E-mail address of N.J. Fisch: fisch@pppl.gov

Subject classification: G0, Te; G0, Tt; G0, Ti; H1, Te; H1, Ti; D1, Te; D1, Tt; D1, Ti; M0 